\title{
A NORM CONVERGENCE RESULT ON RANDOM PRODUCTS OF RELAXED PROJECTIONS IN HILBERT SPACE
}

\author{
H. H. BAUSCHKE
}

\begin{abstract}
Suppose $X$ is a Hilbert space and $C_{1}, \ldots, C_{N}$ are closed convex intersecting subsets with projections $P_{1}, \ldots, P_{N}$. Suppose further $r$ is a mapping from $\mathbb{N}$ onto $\{1, \ldots, N\}$ that assumes every value infinitely often.We prove (a more general version of) the following result:

If the $N$-tuple $\left(C_{1}, \ldots, C_{N}\right)$ is "innately boundedly regular", then the sequence $\left(x_{n}\right)$, defined by

$$
x_{0} \in X \text { arbitrary, } \quad x_{n+1}:=P_{r(n)} x_{n}, \text { for all } n \geq 0,
$$

converges in norm to some point in $\bigcap_{i=1}^{N} C_{i}$.

Examples without the usual assumptions on compactness are given. Methods of this type have been used in areas like computerized tomography and signal processing.
\end{abstract}

\section{INTRODUCTION, FACTS, AND NOTATION}

Numerous problems in mathematics [10] and physical sciences $[9,8,26]$ can be described as follows. Let $X$ be a real Hilbert space and suppose $T_{1}, \ldots, T_{N}$ are pairwise distinct nonexpansive self-mappings of some closed convex nonempty subset $D$ of $X$; recall that a self-mapping $T$ of $D$ is called nonexpansive, if $\|T x-T y\| \leq\|x-y\|$, for all $x, y \in D$. Suppose further that the set of fuxed points, Fix $T_{i}:=\left\{x \in D: T_{i} x=x\right\}$, of each mapping $T_{i}$ is nonempty and that $C:=\bigcap_{i=1}^{N}$ Fix $T_{i} \neq \varnothing$. The aim is to find such a common fixed point. One frequently employed approach is the following:

Let $r$ be a random mapping for $\{1, \ldots, N\}$, i.e., a surjective mapping from $\mathbb{N}$ onto $\{1, \ldots, N\}$ that takes each value in $\{1, \ldots, N\}$ infinitely often. Then generate a random sequence $\left(x_{n}\right)$ by

$$
x_{0} \in D \text { arbitrary }, \quad x_{n+1}:=T_{r(n)} x_{n}, \quad \text { for all } n \geq 0,
$$

Received by the editors June 23, 1993 and, in revised form, January 24, 1994; originally communicated to the Proceedings of the AMS by Palle E. T. Jorgensen.

1991 Mathematics Subject Classification. Primary 47H09; Secondary 46C99, 47N10, 65F10, 65J05, 65K05, 90C25, 92C55.

Key words and phrases. Banach contraction, computerized tomography, convex feasibility problem, convex programming, convex set, Fejér monotone sequence, Hilbert space, image reconstruction, image recovery, innate bounded regularity, Kaczmarz's method, nonexpansive mapping, orthogonal projection, projection algorithm, projection method, projective mapping, random product, relaxation method, relaxed projection, signal processing, unrestricted iteration, unrestricted product. 
and hope that this sequence converges to some point in $C$. We also speak of a random or unrestricted product (resp. iteration). (For products generated by some form of control, there are many results: for instance, cyclic control arises when $r(n)=n+1 \bmod N$; see [6].)

This is, in general, a hopeless undertaking, as the example $X:=\mathbb{R}, N:=1$, and $T_{1}:=-I$ shows (as usual, $I$ denotes the identity).

So let us temporarily consider the important special case when $D=X$ and each mapping $T_{i}$ is the projection onto some closed convex nonempty subset $C_{i}$ of $X$; hence Fix $T_{i}=C_{i}$. The problem of finding a common fixed point is then the famous Convex Feasibility Problem. This situation allows us to compare the following known results (in fact, all authors listed below have established (much) more general but less comparable results):

Amemiya and Ando [3]: If each set $C_{i}$ is a closed subspace, then the random product converges weakly to the projection onto $C$.

Bruck [7]: If some set $C_{i}$ is compact, then the random product converges in norm to some point in $C$. If $N=3$ and each set $C_{i}$ is symmetric, then the random product converges weakly to some point in $C$.

Dye [11]: If the sets $C_{i}$ are finite-dimensional subspaces, then the random product converges in norm to some point in $C$.

Dye and Reich [14]: If the sets $C_{i}$ have a common "weak internal point" or if $N=3$, then the random product converges weakly to some point in $C$.

Youla [29]: If the sets $C_{i}$ have a common "inner point", then the random product converges weakly to some point in $C$.

Aharoni and Censor [2], Fläm and Zowe [16], Tseng [27], Elsner et al. [15]: If $X$ is finite dimensional, then the random product converges in norm to some point in $C$.

The objective of this paper is to provide a new applicable condition which guarantees norm convergent random products.

The paper is organized as follows: In Section 2, we discuss four important concepts: (innate) bounded regularity is a crucial geometric property of tuples of closed convex sets. Fejér monotonicity and Baillon and Bruck's quasi-projection capture essential properties of random sequences. Relaxed projections and $\mathrm{Ba}-$ nach contractions are subsumed in the class of projective mappings. The third sections contains our main result and some examples.

Suppose $C$ is a closed convex nonempty subset of $X$. The projection onto $C$, denoted $P_{C}$, is the mapping which sends every point to its nearest point in $C$. The associated distance function is defined by $d(\cdot, C): X \rightarrow[0,+\infty[: x \mapsto$ $\left\|x-P_{C} x\right\|=\inf _{c \in C}\|x-c\|$. If $\left.\alpha \in\right] 0,2\left[\right.$, then the mapping $R:=(1-\alpha) I+\alpha P_{C}$ is called a relaxed projection. For $R$, the following holds:

Facts 1.1. (i) [18] $R$ is nonexpansive. (ii) [6, Lemma 2.4(iv)] For every $x \in X$ and every $c \in C,\|x-c\|^{2}-\|R x-c\|^{2} \geq \alpha(2-\alpha)\left\|x-P_{C} x\right\|^{2}$.

A nonexpansive self-mapping $T$ of some closed convex nonempty subset $D$ of $X$ is called a Banach contraction if there is some contraction constant $\kappa \in[0,1[$, such that $\|T x-T y\| \leq \kappa\|x-y\|$, for all $x, y \in D$.

Fact 1.2. [30, Lemma A2]. Suppose $T$ is a nonexpansive self-mapping of some closed convex nonempty subset $D$ of $X$. If $T$ has fixed points and $C$ is 
a closed convex nonempty subset of Fix $T$, then $\|x-T x\| \leq 2 d(x, C)$, for every $x \in D$.

Finally, " $\rightarrow$ " abbreviates norm converge and "int" stands for the interior.

\section{FOUR HANDY TOOLS}

Definition 2.1 (Tool 1: (innate) bounded regularity). An $N$-tuple $\left(C_{1}, \ldots, C_{N}\right)$ of closed convex intersecting sets is called boundedly regular if for every bounded sequence $\left(x_{n}\right)$ in $X$,

$\max \left\{d\left(x_{n}, C_{i}\right): i \in\{1, \ldots, N\}\right\} \rightarrow 0$ implies $d\left(x_{n}, C_{1} \cap \cdots \cap C_{N}\right) \rightarrow 0$.

We say that $\left(C_{1}, \ldots, C_{N}\right)$ is innately boundedly regular if $\left(C_{j}\right)_{j \in J}$ is boundedly regular, for every nonempty subset $J$ of $\{1, \ldots, N\}$.

Facts 2.2. Suppose $C_{1}, \ldots, C_{N}$ are closed convex intersecting sets in $X$. Then the $N$-tuple $\left(C_{1}, \ldots, C_{N}\right)$ is innately boundedly regular, whenever at least one of the following conditions holds:

(i) All sets, except possibly one, are boundedly compact.

(ii) $X$ is finite dimensional.

(iii) Each set is a closed subspace and the sum $\sum_{j \in J} C_{j}^{\perp}$ is closed, for every nonempty subset $J$ of $\{1, \ldots, N\}$.

(iv) Each set is a closed subspace and all sets, except possibly one, are finite dimensional.

(v) Each set is a closed subspace and all sets, except possibly one, have finite codimension.

(vi) Each set is a polyhedron, i.e. a finite intersection of half-spaces.

(vii) Each set is a hyperplane.

(viii) Each set is a half-space.

(ix) There is some $i \in\{1, \ldots, N\}$ such that $C_{i} \cap \bigcap_{j \in\{1, \ldots, N\} \backslash\{i\}}$ int $C_{j} \neq \varnothing$.

Proof. (i), (ii), .. , (ix), follow from [6, Proposition 5.4(i), Proposition 5.4(iii), Theorem 5.19, Corollary 5.21(i), Corollary 5.21(ii), Corollary 5.26, Corollary 5.22, Fact 5.23, Corollary 5.14], respectively.

Definition 2.3 (Tool 2: Fejér monotone sequences). Suppose $C$ is a closed convex nonempty subset of $X$ and $\left(x_{n}\right)$ is a sequence in $X$. We say that $\left(x_{n}\right)$ is Fejér monotone w.r.t. $C$ if

$$
\left\|x_{n+1}-c\right\| \leq\left\|x_{n}-c\right\|, \quad \text { for every } c \in C \text { and all } n \text {. }
$$

Facts 2.4. Suppose the sequence $\left(x_{n}\right)$ is Fejér monotone w.r.t. some closed convex nonempty subset $C$ of $X$. Then (see [20] or [6]):

(i) The sequences $\left(d\left(x_{n}, C\right)\right),\left(\left\|x_{n}-c\right\|\right)$ are decreasing and convergent for every $c \in C$. In particular, $\left(x_{n}\right)$ is bounded.

(ii) $\left(x_{n}\right)$ converges in norm to some point in $C$ if and only if there is some subsequence $\left(x_{n_{k}}\right)$ of $\left(x_{n}\right)$ with $d\left(x_{n_{k}}, C\right) \rightarrow 0$.

Definition 2.5 (Tool 3: Baillon and Bruck's [4] quasi-projection). Suppose $C$ is a closed convex nonempty subset of $X$ and $x_{0}$ is a point in $X$. The quasiprojection of $x_{0}$ onto $C$, denoted $\mathscr{Q}_{C} x_{0}$, is defined by

$$
\mathscr{Q}_{C} x_{0}:=\left\{x \in C:\|x-c\| \leq\left\|x_{0}-c\right\| \text {, for every } c \in C\right\} .
$$


Proposition 2.6. Suppose $C$ is a closed convex nonempty subset of $X$ and $x_{0}$ is a point in $X$. Then:

(i) $\mathscr{Q}_{C} x_{0}$ is a bounded closed convex nonempty subset of $C$.

(ii) $P_{C} x_{0} \in \mathscr{Q}_{C} x_{0} \subseteq\left\{x \in C:\left\|x-P_{C} x_{0}\right\| \leq d\left(x_{0}, C\right)\right\}$.

(iii) If $x_{0} \in C$, then $\mathscr{Q}_{C} x_{0}=\left\{P_{C} x_{0}\right\}=\left\{x_{0}\right\}$.

(iv) $\mathscr{Q}_{C+z} x_{0}=z+\mathscr{Q}_{C}\left(x_{0}-z\right)$, for every $z \in X$.

(v) If $C$ is a closed affine subspace, then $\mathscr{Q}_{C} \equiv P_{C}$.

(vi) If $\left(x_{n}\right)_{n \geq 0}$ is a Fejér monotone sequence w.r.t. $C$ converging weakly to some point $x \in C$, then $x \in \mathscr{Q}_{C} x_{0}$.

Proof. It is a straightforward to check (i)-(iv).

(v): In view of (iv), we need only consider the case when $C$ is a closed subspace. Pick $\bar{c} \in \mathscr{Q}_{C} x_{0}$, fix an arbitrary real number $t$, and let $c:=P_{C} x_{0}+$ $t\left(\bar{c}-P_{C} x_{0}\right)$. Then $c \in C$ and $\|\bar{c}-c\| \leq\left\|x_{0}-c\right\|$. Squaring yields $(1-t)^{2} \| P_{C} x_{0}-$ $\bar{c}\left\|^{2} \leq\right\| P_{C^{\perp}} x_{0}\left\|^{2}+t^{2}\right\| P_{C} x_{0}-\bar{c} \|^{2}$ or $\left\|P_{C} x_{0}-\bar{c}\right\|^{2}-2 t\left\|P_{C} x_{0}-\bar{c}\right\|^{2} \leq\left\|P_{C^{\perp}} x_{0}\right\|^{2}$. Then letting $t \rightarrow-\infty$, we obtain a contradiction-unless $\bar{c}=P_{C} x_{0}$.

(vi) follows readily from the weak lower semicontinuity of the norm.

Definition 2.7 (Tool 4: projective mappings). Suppose $T$ is a nonexpansive self-mapping of some closed convex nonempty subset $D$ of $X$. We say that $T$ is projective w.r.t. $c$ if $c \in$ Fix $T$ and if for every bounded sequence $\left(x_{n}\right)$ in $D$,

$$
\left\|x_{n}-c\right\|-\left\|T x_{n}-c\right\| \rightarrow 0 \text { implies } d\left(x_{n}, \text { Fix } T\right) \rightarrow 0 .
$$

If $T$ has fixed points and is projective w.r.t. every one of them, then we simply speak of a projective mapping.

Lemma 2.8. Suppose $T$ is projective w.r.t. c. Then:

(i) If $T$ is projective, then $T$ is attracting [6] (see also [25, Corollary 1.1]), i.e., $\|T x-c\|<\|x-c\|$, for every $x \in D \backslash$ Fix $T$ and every $c \in$ Fix $T$.

(ii) $T$ has condition (S) w.r.t. $c$ [12]; i.e., if $\left(x_{n}\right)$ is a bounded sequence in $D$ and $\left\|x_{n}-c\right\|-\left\|T x_{n}-c\right\| \rightarrow 0$, then $x_{n}-T x_{n} \rightarrow 0$.

(iii) For every $x_{0} \in D$, the sequence of iterates $\left(T^{n} x_{0}\right)_{n \geq 0}$ converges in norm to some fixed point of $T$.

Proof. (i) follows easily from the definitions. (ii) follows from Fact 1.2(iii): $\left(T^{n} x_{0}\right)$ is Fejér monotone w.r.t. Fix $T$ and $\left\|T^{n} x_{0}-c\right\|-\left\|T^{n+1} x_{0}-c\right\| \rightarrow 0$, for every $c \in$ Fix $T$; thus $d\left(T^{n} x_{0}\right.$, Fix $\left.T\right) \rightarrow 0$ and the result follows from Facts 2.4 .

Remarks 2.9. (1) Suppose $T$ is a nonexpansive self-mapping of some closed convex nonempty subset $D$ of $X$ with Fix $T \neq \varnothing$. The following condition appears in Petryshyn and Williamson's [25, Theorem 1.2] and [24, Proposition 1]: (PW) For every bounded sequence $\left(x_{n}\right)$ in $D, x_{n}-T x_{n} \rightarrow 0$ implies $d\left(x_{n}\right.$, Fix $\left.T\right) \rightarrow 0$. Clearly, if $T$ is projective w.r.t. some fixed point, then $T$ satisfies condition (PW). In contrast, let $X=D=\mathbb{R}$ and $T x=1-x$. This mapping satisfies condition (PW) and the sequence $\left(T^{n} x\right)$ does not converge, for every $x \notin$ Fix $T=\left\{\frac{1}{2}\right\}$; thus $T$ is not projective w.r.t. $\frac{1}{2}$.

(2) If $T: l_{2} \rightarrow l_{2}: x=\left(x_{1}, x_{2}, \ldots\right) \mapsto\left(x_{2}, x_{3}, \ldots\right)$, then the sequence $\left(T^{n} x\right)$ converges in norm to the (only) fixed point 0 , for every $x \in l_{2}$. However, $T$ is not projective w.r.t. 0 (consider the sequence of unit vectors); hence the converse of (iii) does not hold in general. 
Theorem 2.10. The class of projective mappings includes (i) Banach contractions and (ii) relaxed projections.

Proof. (i) If $\kappa<1$ is a contraction constant for a Banach contraction $T$ and $\{c\}=$ Fix $T$, then $\|T x-c\| \leq \kappa\|x-c\|$ and hence (i) follows from $d^{2}(x$, Fix $T)=\|x-c\|^{2} \leq\left(\|x-c\|^{2}-\|T x-c\|^{2}\right) /\left(1-\kappa^{2}\right)$, for every $x \in D$.

(ii) If $\alpha \in] 0,2\left[\right.$ and $T=(1-\alpha) I+\alpha P$, where $P=P_{C}$ is the projection onto some closed convex nonempty set $C$ in $D=X$, then (Facts 1.1$)\|x-P x\|^{2}=$ $d^{2}(x$, Fix $T) \leq\left(\|x-c\|^{2}-\|T x-c\|^{2}\right) /(\alpha(2-\alpha))$, for every $x \in X$ and every $c \in C$ :

Example 2.11. Genel and Lindenstrauss [17] constructed a firmly nonexpansive (see [18] or [19, Section 11]) self-mapping $T$ of $X:=l_{2}$ and some point $x_{0} \in X$ such that $0 \in$ Fix $T,\left(T^{n} x_{0}\right)_{n \geq 0}$ converges weakly to 0 but not in norm: $\inf _{n}\left\|T^{n} x_{0}\right\|>0$. Therefore, by Lemma 2.8(iii), $T$ is not projective.

Remark 2.12. Using Lemma 2.8(i) and [6, Proposition 2.10], one can show the following: Suppose $D$ is a closed convex nonempty subset of $X$ and $T_{1}, T_{2}$ are projective self-mappings of $D$. If the pair (Fix $T_{1}$, Fix $T_{2}$ ) is boundedly regular, then $T_{2} T_{1}$ is projective.

Example 2.13. On the real line, let $T x=\frac{1}{2}|x|^{2}$, if $|x| \leq 1$ and $T x=|x|-\frac{1}{2}$ otherwise. Then $T$ is projective but does not belong to any of the standard classes of "nice" nonexpansive mappings (cf. [6, Example 2.3]).

\section{THE MAIN RESULT}

Hypothesis. From now on, we always assume that $D$ is a closed convex nonempty subset of $X$, that $N \geq 1$, that $T_{1}, \ldots, T_{N}: D \rightarrow D$ are pairwise distinct and projective w.r.t. some common fixed point $c \in C:=\bigcap_{i=1}^{N} C_{i}$, where each $C_{i}$ equals Fix $T_{i}$, and that the $N$-tuple $\left(C_{1}, \ldots, C_{N}\right)$ is innately boundedly regular.

Definition 3.1. A mapping $T: D \rightarrow D$ is called a full word, denoted $T \in \mathscr{F}:=$ $\mathscr{F}\left(T_{1}, \ldots, T_{N}\right)$, if $T$ can be written as a finite product of the mappings in $\left\{T_{1}, \ldots, T_{N}\right\}$, where each mapping $T_{i}$ occurs at least once. We say that $T$ is an $M$-word, denoted $T \in \mathscr{W}_{M}:=\mathscr{W}_{M}\left(T_{1}, \ldots, T_{N}\right)$, if $T$ can be written as a finite product where at most $M$ different factors $T_{i_{1}}, \ldots, T_{i_{M}}$ occur, for some $M \in\{1, \ldots, N\}$ and some subset $\left\{i_{1}, \ldots, i_{M}\right\}$ of $\{1, \ldots, N\}$.

Note that the identity (the product with 0 factors) is always in $\mathscr{W}_{M}$ and that $\mathscr{F} \subseteq \mathscr{W}_{N}$.

Proposition 3.2. In addition to the hypothesis, suppose that $\left(x_{n}\right)$ is a bounded sequence in $D$, that $\left(W_{n}\right)$ is a sequence in $\mathscr{W}_{N}$, and that $\left\|x_{n}-c\right\|-\left\|W_{n} x_{n}-c\right\| \rightarrow$ 0 . Then $(*) x_{n}-W_{n} x_{n} \rightarrow 0$. Moreover, if each $W_{n} \in \mathscr{F}$, then $d\left(x_{n}, C\right) \rightarrow 0$. Proof. We assume without loss of generality that $c=0$ (otherwise, we translate). For $M \in\{1, \ldots, N\}$ define the statement $(*, M)$ by

For every bounded sequence $\left(x_{n}\right)$ in $D$ and every sequence of words $\left(W_{n}\right)$ in $\mathscr{W}_{M}:$ if $\left\|x_{n}\right\|-\left\|W_{n} x_{n}\right\| \rightarrow 0$, then $x_{n}-W_{n} x_{n} \rightarrow 0$.

Hence, the main statement, $(*)$, holds exactly when $(*, N)$ does. 
Step 1. $(*, 1)$ holds. Otherwise, there is a bounded sequence $\left(x_{n}\right)$ in $D$, a sequence of words $\left(W_{n}\right)$ in $\mathscr{W}_{1}$, some $i \in\{1, \ldots, N\}$, and a sequence of (strictly) positive integers $\left(l_{n}\right)$ such that $\left\|x_{n}\right\|-\left\|W_{n} x_{n}\right\| \rightarrow 0, \inf _{n} \| x_{n}-$ $W_{n} x_{n} \|>0$, and $W_{n}=T_{i}^{l_{n}}$, for all $n$. Now $\left\|x_{n}\right\| \geq\left\|T_{i} x_{n}\right\| \geq\left\|W_{n} x_{n}\right\|$, hence $\left\|x_{n}\right\|-\left\|T_{i} x_{n}\right\| \rightarrow 0$. Because $T_{i}$ is projective w.r.t. $c=0$, we conclude (Fact $1.2)$

$$
0 \leftarrow d\left(x_{n}, C_{i}\right)=d\left(x_{n}, \text { Fix } T_{i}\right) \geq d\left(x_{n}, \text { Fix } T_{i}^{l_{n}}\right) \geq \frac{1}{2}\left\|x_{n}-W_{n} x_{n}\right\|,
$$

which contradicts $\inf _{n}\left\|x_{n}-W_{n} x_{n}\right\|>0$. Hence Step 1 is verified.

Step 2. If $M \in\{2, \ldots, N\}$ and $(*, M-1)$ holds, then so does $(*, M)$. Otherwise, there is a bounded sequence $\left(x_{n}\right)$ in $D$, a sequence of words $\left(W_{n}\right)$ in $\mathscr{W}_{M} \backslash \mathscr{W}_{M-1}$, and some indices $\left\{i_{1}, \ldots, i_{M}\right\} \subseteq\{1, \ldots, N\}$ such that $W_{n} \in$ $\mathscr{F}\left(T_{i_{1}}, \ldots, T_{i_{M}}\right)$, for all $n$, and $\left\|x_{n}\right\|-\left\|W_{n} x_{n}\right\| \rightarrow 0$, but $\inf _{n}\left\|x_{n}-W_{n} x_{n}\right\|>0$. Fix $m \in\{1, \ldots, M\}$ and write $W_{n}=L_{n} T_{i_{m}} R_{n}$, where $R_{n} \in \mathscr{W}_{M-1}$, for all $n$. Since $\left\|x_{n}\right\| \geq\left\|R_{n} x_{n}\right\| \geq\left\|T_{i_{m}} R_{n} x_{n}\right\| \geq\left\|W_{n} x_{n}\right\|$, we get (i) $\left\|x_{n}\right\|-\left\|R_{n} x_{n}\right\| \rightarrow 0$ and (ii) $\left\|R_{n} x_{n}\right\|-\left\|T_{i_{m}} R_{n} x_{n}\right\| \rightarrow 0$. The fact that $(*, M-1)$ holds and (i) imply $x_{n}-R_{n} x_{n} \rightarrow 0$; thus

$$
T_{i_{m}} R_{n} x_{n}-T_{i_{m}} x_{n} \rightarrow 0,
$$

by nonexpansivity of $T_{i_{m}}$. Since $T_{i_{m}}$ is projective w.r.t. $c=0$, (ii) and Lemma 2.8(ii) yield

$$
R_{n} x_{n}-T_{i_{m}} R_{n} x_{n} \rightarrow 0 .
$$

Adding the three preceding sequences gives $x_{n}-T_{i_{m}} x_{n} \rightarrow 0$; hence $\left\|x_{n}\right\|-$ $\left\|T_{i_{m}} x_{n}\right\| \rightarrow 0$ and thus $d\left(x_{n}, C_{i_{m}}\right) \rightarrow 0$. Because $m$ has been chosen arbitrarily, we conclude $\max _{m \in\{1, \ldots, M\}} d\left(x_{n}, C_{i_{m}}\right) \rightarrow 0$, and further (the $M$-tuple $\left(C_{i_{m}}\right)_{m \in\{1, \ldots, M\}}$ is boundedly regular $) d\left(x_{n}, \cap_{m=1}^{M} C_{i_{m}}\right) \rightarrow 0$. Hence, by Fact $1.2,0 \leftarrow d\left(x_{n}, \cap_{m=1}^{M} C_{i_{m}}\right) \geq d\left(x_{n}\right.$, Fix $\left.W_{n}\right) \geq \frac{1}{2}\left\|x_{n}-W_{n} x_{n}\right\|$ which is the desired contradiction. Therefore, Step 2 is also verified.

Conclusion. (*) holds.

Step 3. The "Moreover" part. Assume to the contrary that the "Moreover" part is wrong. Then there is some bounded sequence $\left(x_{n}\right)$ in $D$ and a sequence of full words $\left(W_{n}\right)$ in $\mathscr{F}$ such that $\left\|x_{n}\right\|-\left\|W_{n} x_{n}\right\| \rightarrow 0$, but $\inf _{n} d\left(x_{n}, C\right)>0$. Analogously to Step 2, we deduce $d\left(x_{n}, C\right) \rightarrow 0$, which is absurd.

Condition $(*)$ also appears as Dye and Reich's semigroup condition $(\mathbf{S})$ in [13]. We are now ready for the main result:

Theorem 3.3. In addition to the hypothesis, suppose $r$ is a random mapping for $\{1, \ldots, N\}$. Then the random sequence $\left(x_{n}\right)$, defined by

$$
x_{0} \in D \text { arbitrary, } \quad x_{n+1}:=T_{r(n)} x_{n}, \quad \text { for all } n \geq 0,
$$


Proof. Since $r$ is a random mapping, we can find a subsequence $\left(n_{k}\right)_{k}$ of $(n)_{n}$ such that $W_{k}:=T_{r\left(n_{k+1}-1\right)} \cdots T_{r\left(n_{k}\right)} \in \mathscr{F}$, for all $k$. The sequence $\left(x_{n_{k}}\right)$ is Fejér monotone w.r.t. $C$ and the sequence $\left(\left\|x_{n_{k}}-c\right\|\right)$ converges; thus, by the last proposition, $d\left(x_{n_{k}}, C\right) \rightarrow 0$. On the other hand, $\left(x_{n_{k}}\right)$ is a subsequence of $\left(x_{n}\right)$; therefore, the result follows from Facts 2.4(ii) and Proposition 2.6(vi).

The reader may deduce a variety of examples by putting together Facts 2.2 , Proposition 2.6, Theorem 2.10, and Theorem 3.3; here, we give a rather small selection.

Example 3.4 ("Random Kaczmarz"). Suppose each set $C_{i}$ is a hyperplane. Then the random product of relaxed projections onto these hyperplanes converges in norm to the projection onto $C$.

Remark 3.5. The cyclic control version with unrelaxed projections in Euclidean space was already known to Kaczmarz [22] in 1937.

Example 3.6 ("Random Agmon/Motzkin \& Schoenberg"). If each set $C_{i}$ is a half-space, then the random product of relaxed projections converges in norm to some point in $\mathscr{Q}_{C} x_{0}$.

Remark 3.7. The cyclic control version is due to Gubin et al. [20], whereas the "remotest set control" version is due to Agmon [1] and to Motzkin and Schoenberg [23]. In the field of image reconstruction, these methods are known as "AMS relaxation methods" or "ART for inequalities" $[9,8]$.

Example 3.8 ("Random von Neumann/Halperin"). Suppose each set $C_{i}$ is a closed subspace and $\sum_{j \in J} C_{j}^{\perp}$ is closed, for every nonempty subset $J$ of $\{1, \ldots, N\}$. Then the random product of relaxed projections onto the subspaces $C_{i}$ converges in norm to the projection onto $C$.

Remark 3.9. The cyclic control version is due to von Neumann [28] and to Halperin [21] and does not require the assumption on the closedness of the sum of the complements; see also Deutsch's survey [10] for applications and Baillon et al.'s [5, Corollary 2.4] for a more general (nonlinear) result.

\section{ACKNOWLEDGMENT}

The author wishes to thank Jon Borwein for numerous discussions. Thanks are also due to two anonymous referees for many useful suggestions including drawing my attention to references $[5,12,13,19,24,25]$ which led to an improved version of the original manuscript.

\section{REFERENCES}

1. S. Agmon, The relaxation method for linear inequalities, Canad. J. Math. 6 (1954), 382-392.

2. R. Aharoni and Y. Censor, Block-iterative projection methods for parallel computation of solutions to convex feasibility problems, Linear Algebra Appl. 120 (1989), 165-175.

3. I. Amemiya and T. Ando, Convergence of random products of contractions in Hilbert space, Acta Sci. Math. (Szeged) 26 (1965), 239-244.

4. J. B. Baillon and R. E. Bruck, Ergodic theorems and the asymptotic behavior of contraction semigroups, Fixed Point Theory and Applications (K. T. Tan, ed.), Proc. Internat. Conf., Halifax, Nova Scotia, Canada, June 9-14, 1991, World Scientific Publ., Singapore, 1992, pp. 12-26. 
5. J. B. Baillon, R. E. Bruck, and S. Reich, On the asymptotic behaviour of nonexpansive mappings and semigroups in Banach spaces, Houston J. Math. 4 (1978), 1-9.

6. H. H. Bauschke and J. M. Borwein, On projection algorithms for solving convex feasibility problems, Technical rep., Simon Fraser Univ., 1993.

7. R. E. Bruck, Random products of contractions in metric and Banach spaces, J. Math. Anal. Appl. 88 (1982), 319-332.

8. Y. Censor, Parallel application of block-iterative methods in medical imaging and radiation therapy, Math. Programming 42 (1988), 307-325.

9. Y. Censor and G. T. Herman, On some optimization techniques in image reconstruction from projections, Appl. Numer. Math. 3 (1987), 365-391.

10. F. Deutsch, The method of alternating orthogonal projections, Approximation Theory, Spline Features and Applications (S. P. Singh, ed.), Proc. Conf., Hotel Villa del Mare, Maratea, Italy, April 28, 1991, May 9, 1991, Kluwer Academic, Amsterdam, 1992, pp. 105-121.

11. J. M. Dye, Convergence of random products of compact contractions in Hilbert space, Integral Equations and Operator Theory 12 (1989), 12-22.

12. J. M. Dye, T. Kuczumow, P.-K. Lin, and S. Reich, Random products of nonexpansive mappings in spaces with the Opial property, (B.-L. Lin and W. B. Johnson, eds.), Banach Spaces, Contemporary Math., vol. 144, Amer. Math. Soc., Providence, RI, 1993, pp. 87-93.

13. J. M. Dye and S. Reich, Random products of nonexpansive mappings, Optimization and Nonlinear Analysis (A. Ioffe, M. Marcus, and S. Reich, eds.), Proc. Binational Workshop on Optimization and Nonlinear Analysis, Technion City, Haifa, 21-27, March 1990, Pitman Res. Notes in Math. Ser., vol. 244, Longman Sci. Tech, Harlow, England, 1992, pp. 106-118.

14. Unrestricted iterations of nonexpansive mappings in Hilbert space, Nonlinear Anal. 18 (1992), 199-207.

15. L. Elsner, I. Koltracht, and M. Neumann, Convergence of sequential and asynchronous nonlinear paracontractions, Numer. Math. 62 (1992), 305-319.

16. S. D. Flàm and J. Zowe, Relaxed outer projections, weighted averages and convex feasibility, BIT 30 (1990), 289-300.

17. A. Genel and J. Lindenstrauss, An example concerning fixed points, Israel J. Math. 22 (1975), $81-86$.

18. K. Goebel and W. A. Kirk, Topics in metric fixed point theory, Cambridge Stud. Adv. Math., vol. 28, Cambridge Univ. Press, Cambridge, 1990.

19. K. Goebel and S. Reich, Uniform convexity, hyperbolic geometry and nonexpansive mappings, Monographs and Textbooks in Pure and Appl. Math., vol. 83, Marcel Dekker, New York, 1984.

20. L. G. Gubin, B. T. Polyak, and E. V. Raik, The method of projections for finding the common point of convex sets, USSR Comput. Math. Math. Phys. 7 (1967), 1-24.

21. I. Halperin, The product of projection operators, Acta Sci. Math. (Szeged) 23 (1962), 96-99.

22. S. Kaczmarz, Angenäherte Auflösung von Systemen linearer Gleichungen, Bull. Internat. Acad Polon. Sci. Lettres. Cl. Sci. Math. Natur. Sér. A: Sci. Math., Imprimerie de l'Université, Cracovie, 1937, pp. 355-357.

23. T. S. Motzkin and I. J. Schoenberg, The relaxation method for linear inequalities, Canad. J. Math. 6 (1954), 393-404.

24. W. V. Petryshyn and T. E. Williamson, Jr., A necessary and sufficient condition for the convergence of a sequence of iterates for quasi-nonexpansive mappings, Bull. Amer. Math. Soc. 78 (1972), 1027-1031.

25. __ Strong and weak convergence of the sequence of successive approximations for quasinonexpansive mappings, J. Math. Anal. Appl. 43 (1973), 459-497.

26. M. I. Sezan, An overview of convex projections theory and its applications to image recovery problems, Ultramicroscopy 40 (1992), 55-67.

27. P. Tseng, On the convergence of the products of firmly nonexpansive mappings, SIAM J. Optim. 2 (1992), 425-434. 
28. J. von Neumann, Functional operators, vol. II. The geometry of orthogonal spaces, Ann. of Math. Stud., no. 22, Princeton Univ. Press, Princeton, NJ, 1950. Reprint of mimeographed lecture notes first distributed in 1933.

29. D. C. Youla, On deterministic convergence of iterations of relaxed projection operators, J. Visual Communication and Image Representation 1 (1990), 12-20.

30. D. C. Youla and $\mathrm{H}$. Webb, Image reconstruction by the method of convex projections: Part 1. Theory, IEEE Trans. Medical Imaging MI-1 (1982), 81-94.

Centre for Experimental and Constructive Mathematics, Simon Fraser University, Burnaby, British Columbia, Canada V5A 1S6

E-mail address: bauschke@cecm.sfu.ca 